\title{
Understanding the Fluorescence of TADF Light-emitting Dyes (Supporting Information)
}

\author{
Georgi Valchanov, ${ }^{\mathrm{a}}$ Anela Ivanova,,${ }^{\mathrm{a}}{ }^{*}$ Alia Tadjer, ${ }^{\mathrm{a}}$ Dennis Chercka, ${ }^{\mathrm{b}}$ Martin Baumgarten ${ }^{\mathrm{b}}$ \\ ${ }^{a}$ Department of Physical Chemistry, Faculty of Chemistry and Pharmacy, University of Sofia, 1 James \\ Bourchier Ave., 1164 Sofia, Bulgaria \\ ${ }^{b}$ Max Planck Institute for Polymer Research, Ackermannweg 10, 55128 Mainz, Germany
}

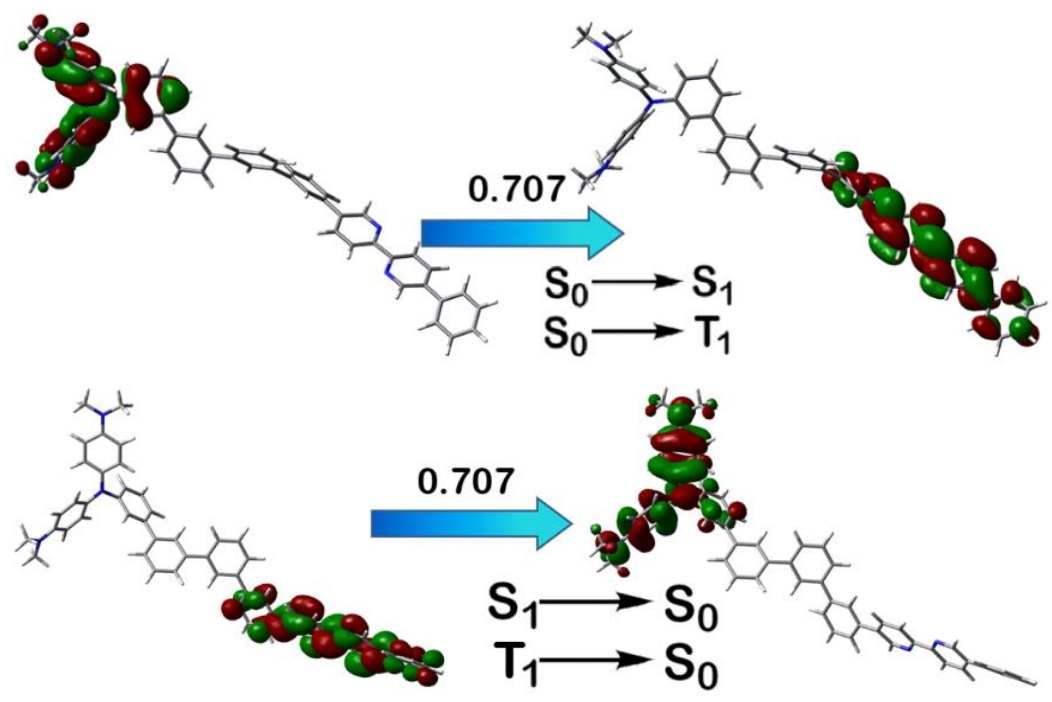

Figure S1. Absorption (top) and emission (bottom) transitions of DMATPhA-BPh-DPhBP; the coefficients of the depicted contribution to the transitions are given. 


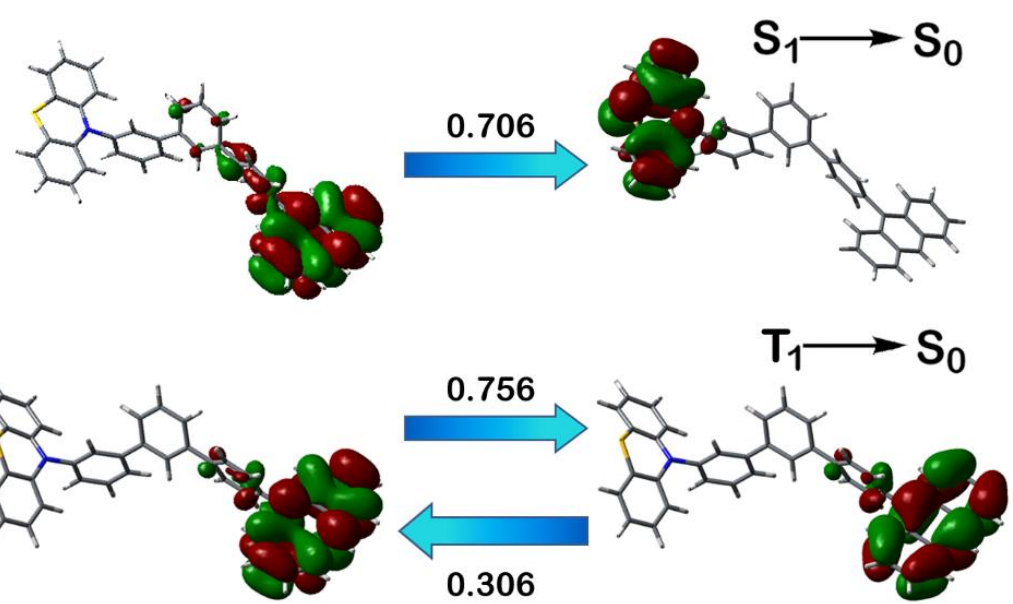

Figure S2. Singlet-singlet (top) and triplet-singlet (bottom) emission transitions of $\mathrm{PhT}-\mathrm{BPh}-$ $\mathrm{PhAnth}$; the coefficients of the depicted contribution to the transitions are given.

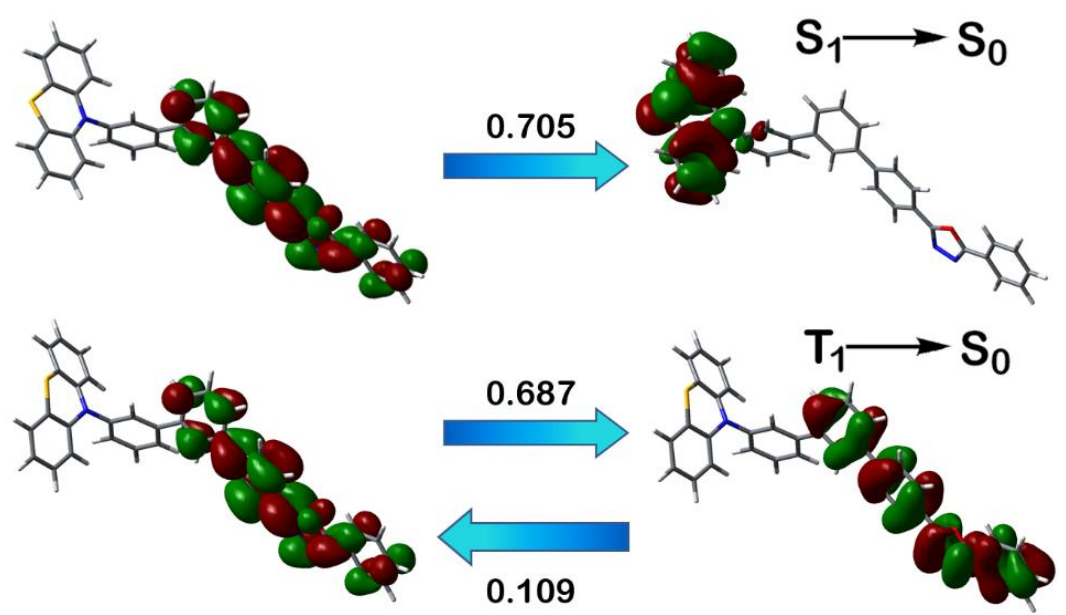

Figure S3. Singlet-singlet (top) and triplet-singlet (bottom) emission transitions of $\mathrm{PhT}-\mathrm{BPh}$ DPhOx; the coefficients of the depicted contribution to the transitions are given.

The computations show that PhT-systems have triplet-singlet transitions involving orbitals positioned mainly on the acceptor moiety (Figures S2, S3 of SI). In both cases with a phenothiazine donor there are re-excitation coefficients in addition to the relaxation ones - a feature, which was observed only for the absorption transitions of the molecule with phenylanthracene acceptor and also in the phenyl-anthracene molecule itself. ${ }^{\mathrm{S} 1}$ This means that there is contribution to the TD-DFT operator of a configuration representing annihilation of an electron in the ground state and its creation in the excited state. This obviously leads to decreasing of the triplet-singlet transition energy, as the two molecules with a phenothiazine donor are the only ones with non-degenerate singlet and triplet excited states. These are also the molecules with the largest Stokes shifts, an explanation of which is given in the main manuscript. 

(A)
$\mathbf{S}_{1} \equiv \mathbf{T}_{1}$
(B) $\mathrm{S}_{0} \quad \mathrm{~S}_{1} \equiv \mathrm{T}_{1}$
(C) $\begin{array}{lll}S_{0} & S_{1} & T_{1}\end{array}$

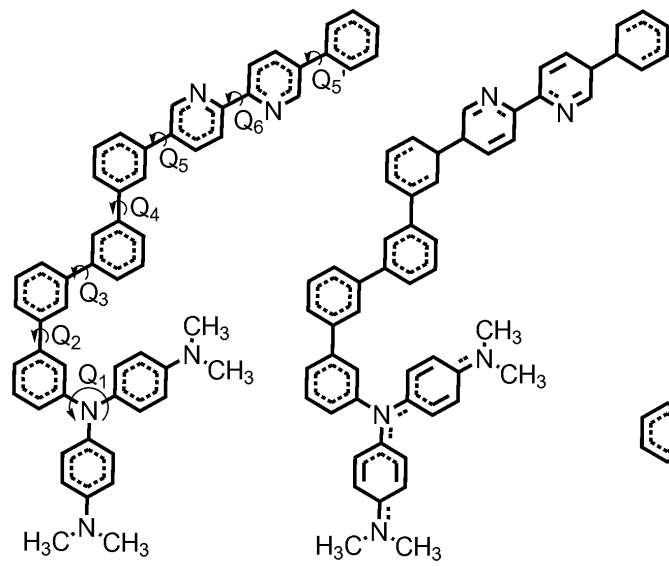

(C)

DMATPhA-BPh-TPh

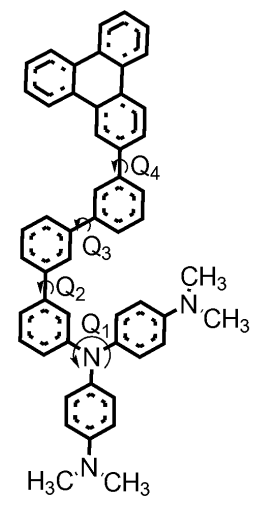

$\mathrm{S}_{1}$

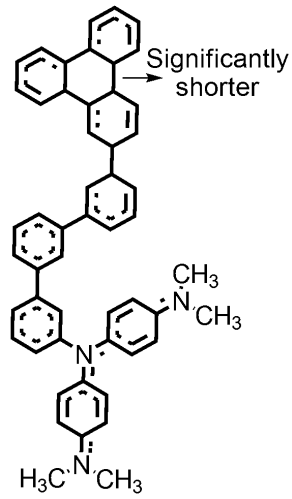

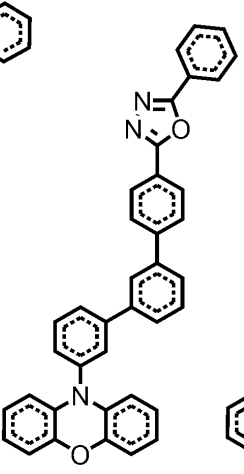

(D)

PhT-BPh-PhA

$\mathrm{S}_{0}$

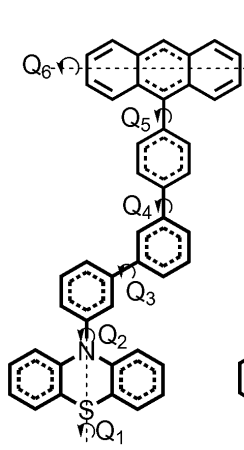

$\mathbf{s}_{1}$

$\mathrm{T}_{1}$

$s_{1}$

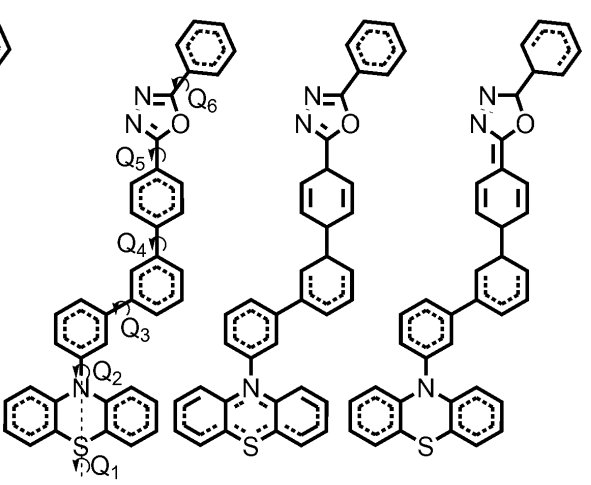
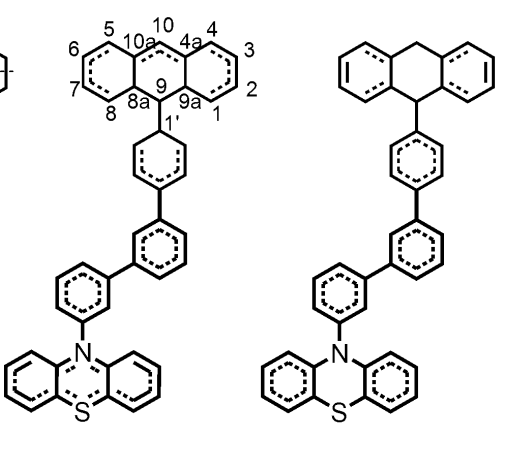

Figure S4. Change in geometry (bond lengths) from ground (left) to the singlet (middle) and triplet (right) excited states of the D-BPh-A molecules; hydrogen atoms are omitted for clarity; the carbon atoms in the anthracene moiety are numbered and the torsion angles corresponding to rotation about single bonds are denoted.

In some of the D-spacer-A molecules the excited donor/acceptor resembles either the singlet or the triplet excited state of the isolated molecule. For instance, the excited DMATPhA within the combined molecules is similar to the isolated excited triplet, whereas $\mathrm{PhOx}$ and $\mathrm{PhT}-$ to their excited singlets. In the two latter molecules, also flattening of the cyclic system takes place upon excitation, adding further to the red shift. Among the acceptors, DPhBP within the large system excites alike to its isolated $\mathrm{T}_{1}$, while the other three molecules undergo unique structural relaxation in the combined molecules. For DPhOx, planarity in all states and more extended conjugation in the vicinity of the nitrogen atoms upon excitation is the cause of red shift and some quinoidization of the bonds results in blue shift. The degree of delocalization enhancement and the quinoidization patterns, however, are different in isolated and bound, and in singlet and triplet state, which leads to dissimilar Stokes shifts. 

(A)
$\mathrm{S}_{0}$
$\mathbf{S}_{1}$
$T_{1}$

PhOx
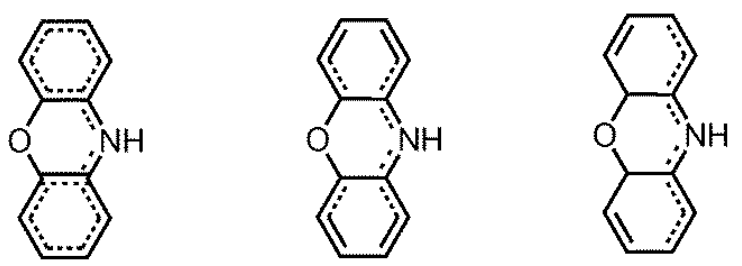

(B)

$\mathbf{S}_{0}$

$\mathbf{S}_{1}$

$\mathrm{T}_{1}$

PhT
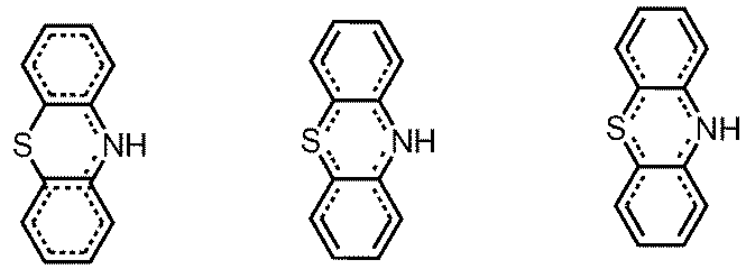

Figure S5. Change in geometry (bond lengths) from ground (left) to the singlet (middle) and triplet (right) excited states of the isolated donors; hydrogen atoms are omitted for clarity. 

(A)
$\mathrm{S}_{0}$
$\mathbf{S}_{1}$
$T_{1}$<smiles></smiles>

(B) $\quad \mathrm{s}_{0}$

$\mathrm{s}_{1}$

$T_{1}$

TPh<smiles>c1ccc2c(c1)c1ccccc1c1ccccc21</smiles>

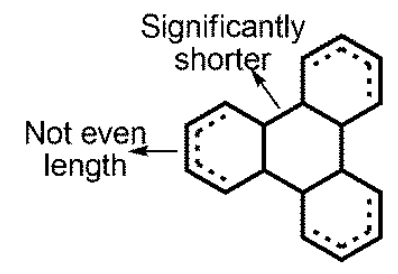<smiles>CC(C)[NH2+]C1=CC2C3C=CC=CC3C3C4C=CC=CC4C4C=CC=CC4C23C=C1</smiles>

(C)

$\mathbf{S}_{1}$

$T_{1}$

\section{PhAnth}
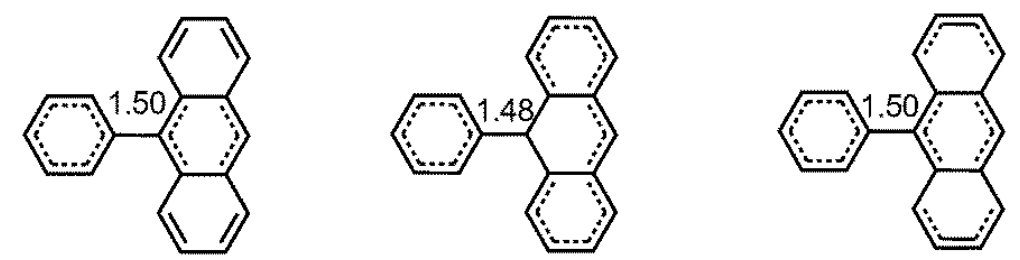

Figure S6. Change in geometry (bond lengths) from ground (left) to the singlet (middle) and triplet (right) excited states of the isolated acceptors; hydrogen atoms are omitted for clarity; the torsion angles corresponding to rotation about single bonds are denoted. 
Table S1. Change in torsion angles (in degree) from the ground state to the first excited singlet or triplet state of the D-BPh-A molecules and of isolated donors or acceptors; $\Delta \Theta=\Theta_{\text {excited state }}$ $\Theta_{\text {ground state }}$ the left number in each cell corresponds to the change $S_{1}-S_{0}$ and the right one - to $T_{1}$ $\mathrm{S}_{0}$ if the two excited states are different.

\begin{tabular}{|c|c|c|c|c|c|c|}
\hline Molecule & $\Delta \Theta_{1}$ & $\Delta \Theta_{2}$ & $\Delta \Theta_{3}$ & $\Delta \Theta_{4}$ & $\Delta \Theta_{5}$ & $\Delta \Theta_{6}$ \\
\hline \multicolumn{7}{|l|}{ D-BPh-A molecules } \\
\hline DMATPhA-BPh-DPhBP & 7.2 & 0.6 & 0.9 & 0.6 & -12.5 & 0.5 \\
\hline DMATPhA-BPh-DPhOx & 7.9 & 0.3 & 0.0 & -14.5 & 0.0 & 0.0 \\
\hline DMATPhA-BPh-TPh & 8.1 & 3.0 & -4.4 & -20.0 & ----- & ---- \\
\hline PhT-BPh-DPhOx & $-36.3 /-0.1$ & $7.9 /-1.1$ & $-0.7 / 1.2$ & $-18.6 /-17.7$ & $0.0 / 0.0$ & $0.0 / 0.0$ \\
\hline $\mathrm{PhT}-\mathrm{BPh}-\mathrm{PhAr}$ & $-35.6 / 0.1$ & $-6.5 /-0.6$ & $-1.2 /-0.8$ & $-3.3 / 0.0$ & $-18 /-7.6$ & $3.3 / 0.5$ \\
\hline PhOx-BPh-DPhOx & 2.0 & 2.2 & -4.4 & -18.3 & 0.0 & 0.0 \\
\hline \multicolumn{7}{|l|}{ Donors } \\
\hline DMATPhA & $38.4 / 1.4$ & ----- & ----- & ----- & ----- & ----- \\
\hline PhT & $-32.8 /-33.2$ & ----- & ----- & ---- & ----- & ----- \\
\hline $\mathrm{PhOx}$ & $-14.1 /-14.0$ & ---- & ----- & ----- & ----- & ---- \\
\hline \multicolumn{7}{|l|}{ Acceptors } \\
\hline DPhBP & ----- & ----- & ----- & ---- & -17.5 & -0.5 \\
\hline DPhOx & ---- & ----- & ---- & ----- & $0.0 / 0.0$ & $0.0 / 0.0$ \\
\hline PhAnth & ----- & ----- & ----- & ---- & $-21.9 / 1.3$ & $4.5 / 0.0$ \\
\hline
\end{tabular}

Table S2. Stokes shifts (in eV) of the longest-wavelength singlet-singlet (S) and singlet-triplet (T) transition of the isolated donors and acceptors

\begin{tabular}{|l|c|c|}
\hline Molecule & Stokes $(\mathbf{S})$ & Stokes $(\mathbf{T})$ \\
\hline DMATPhA & 0.944 & 0.825 \\
\hline PhT & 0.900 & 0.941 \\
\hline PhOx & 0.643 & 0.658 \\
\hline DPhBP & 0.612 & 0.888 \\
\hline DPhOx & 0.637 & 1.043 \\
\hline TPh & 0.269 & 0.210 \\
\hline PhAnth & 0.482 & 0.454 \\
\hline
\end{tabular}


Table S3. Energies of the frontier orbitals (in eV) of the improved models

\begin{tabular}{|l|l|l|l|l|l|}
\hline Donor & Spacer & Acceptor & Attachment & EHOMO & ELUMO $^{\text {Ata }}$ \\
\hline \hline DMATPhA & Fl & DPhOx & m-m-m & -4.293 & -1.629 \\
\hline DMATPhA & DMFl & DPhOx & m-m-m & -4.295 & -1.615 \\
\hline DMATPhA & Fl & DPhOx & m-m'-m & -4.254 & -1.641 \\
\hline DMATPhA & Fl & DPhOx & m-m-p & -4.280 & -1.612 \\
\hline DMATPhA & Fl & DPhOx & m-p-m & -4.249 & -1.640 \\
\hline DMATPhA & Fl & DPhOx & p-m-m & -4.299 & -1.664 \\
\hline DMATPhA & Fl & MPhOx & m-m-p & -4.303 & -1.445 \\
\hline DMATPhA & Fl & OxtBu & m-m-p & -4.298 & -1.065 \\
\hline DMATPhA & Fl & Ox & m-m-p & -4.306 & -1.212 \\
\hline DMADMTPhA & Fl & MPhOx & m-m-p & -4.426 & -1.454 \\
\hline DMADMTPhA & Fl & OxtBu & m-m-p & -4.428 & -1.070 \\
\hline DMADPhA & Fl & MPhOx & m-m-p & -4.309 & -1.411 \\
\hline DPhA & Fl & MPhOx & m-m-p & -5.155 & -1.546 \\
\hline
\end{tabular}


(A)
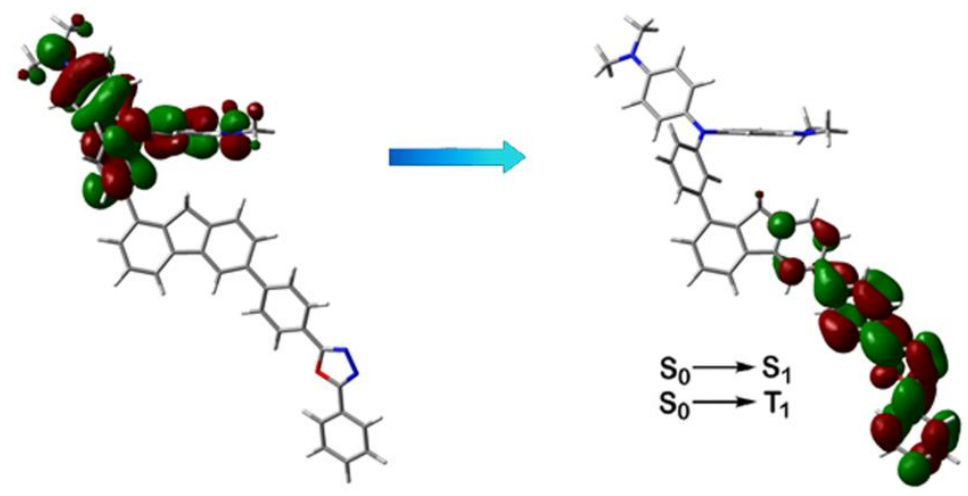

(B)

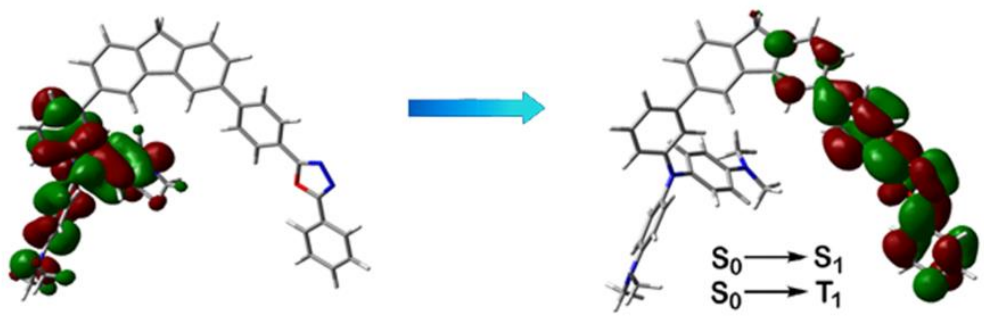

(C)

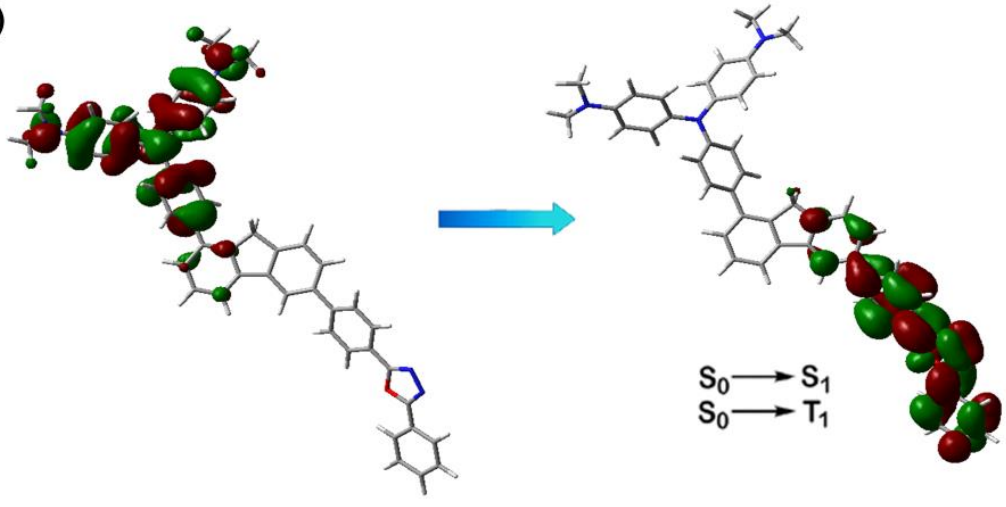

(D)

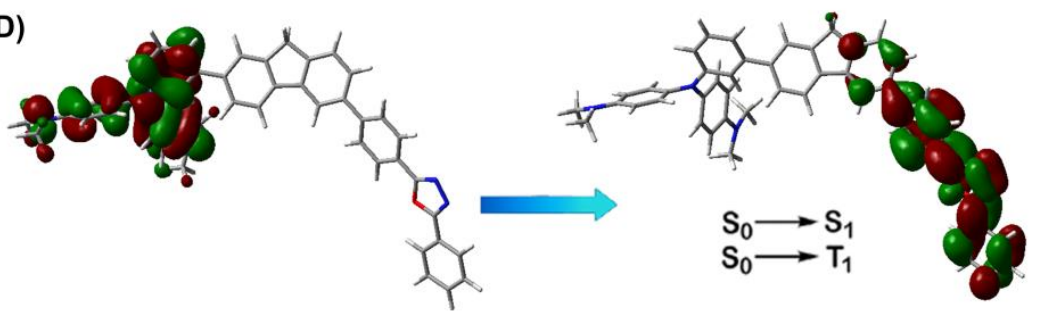

(E)

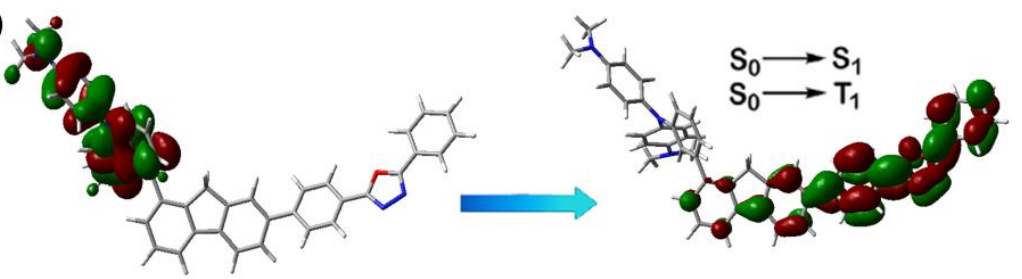

Figure S7. Molecular orbitals taking part in the absorption transitions of the molecule: (A) $\mathrm{m}$ DMATPhA-m,m-Fl-DPhOx; (B) m-DMATPhA-m',m-Fl-DPhOx; (C) p-DMATPhA-m,m-FlDPhOx; (D) m-DMATPhA-p,m-Fl-DPhOx; (E) m-DMATPhA-m,p-Fl-DPhOx 

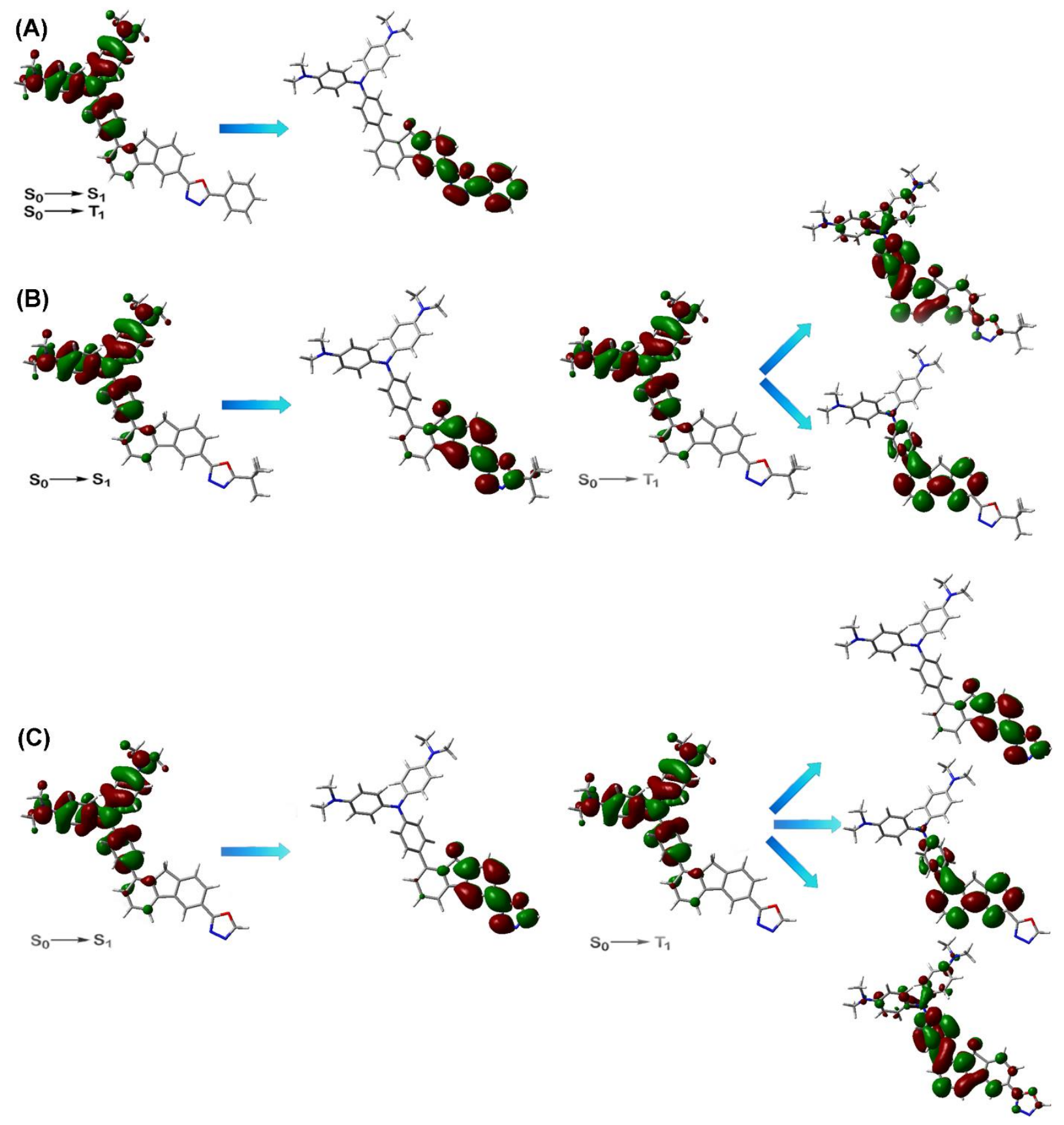

Figure S8. Molecular orbitals taking part in the absorption transitions of the molecule: (A) pDMATPhA-m,m-Fl-MPhOx; (B) p-DMATPhA-m,m-Fl-OxtBu; (C) p-DMATPhA-m,m-Fl-Ox 


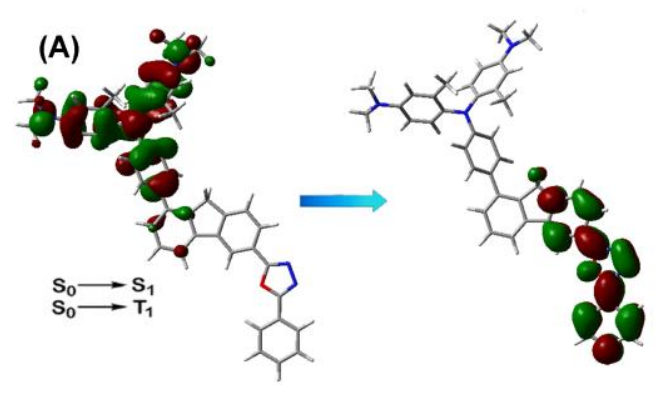

(B)
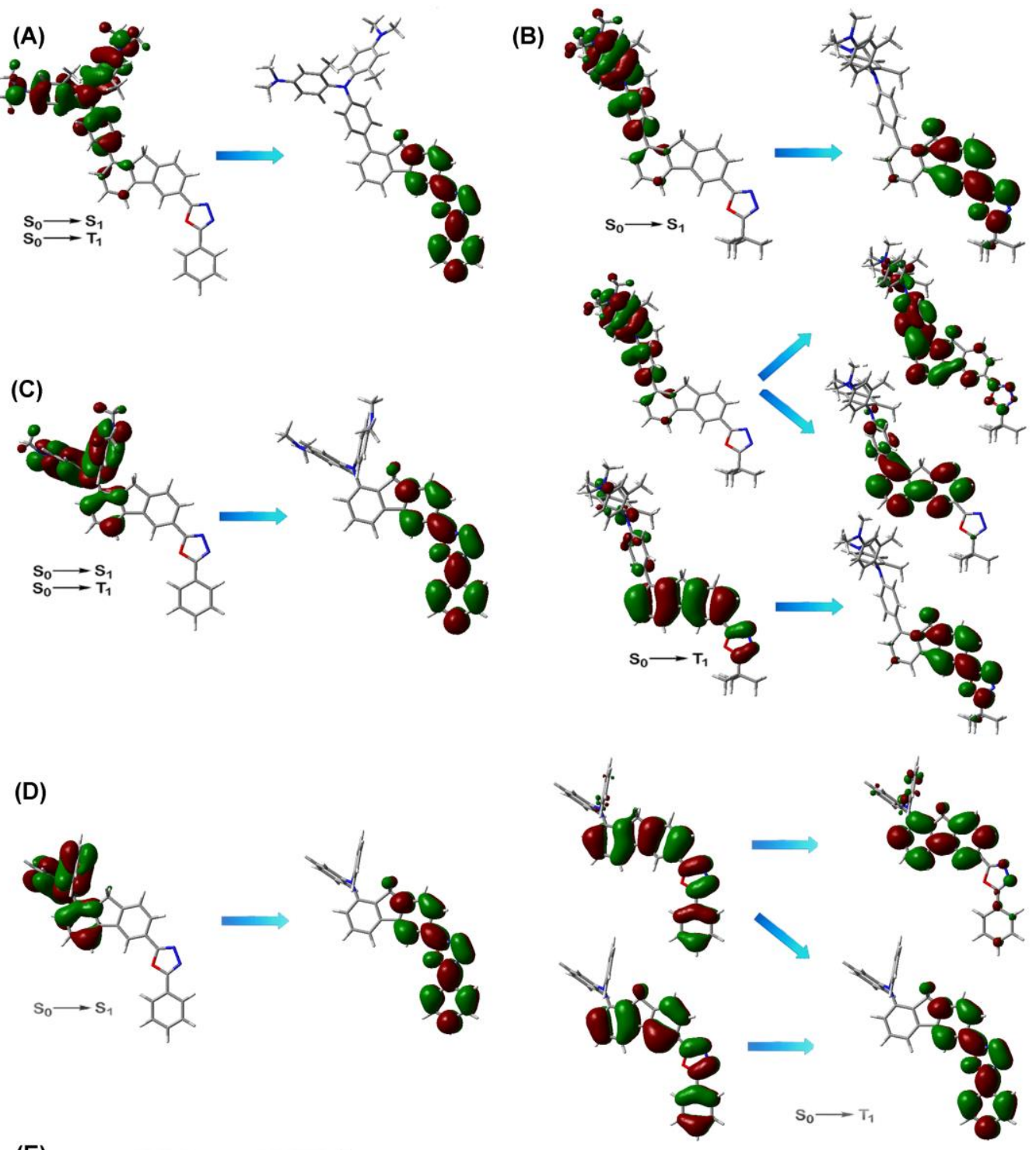

(E)

(D)
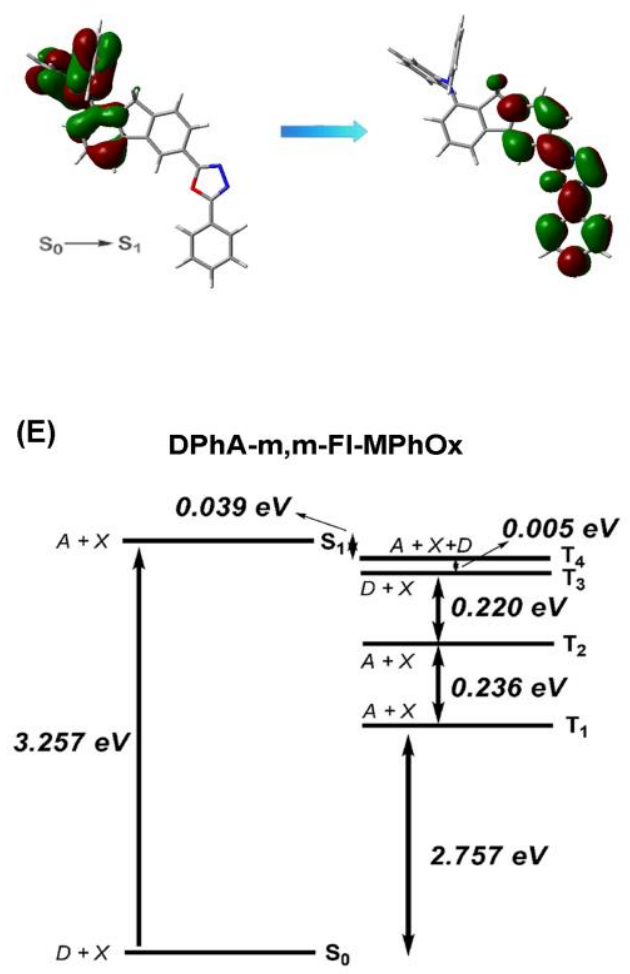

Figure S9. Molecular orbitals taking part in the absorption transitions of the molecule: (A) pDMADMTPhA-m,m-Fl-MPhOx; (B) p-DMADMTPhA-m,m-Fl-OxtBu; (C) p-DMADPhA-m,mFl-MPhOx; (D) DPhA-m,m-Fl-MPhOx; and (E) energy ordering of the excited states in DPhA$\mathrm{m}, \mathrm{m}-\mathrm{Fl}-\mathrm{MPhOx}$ 

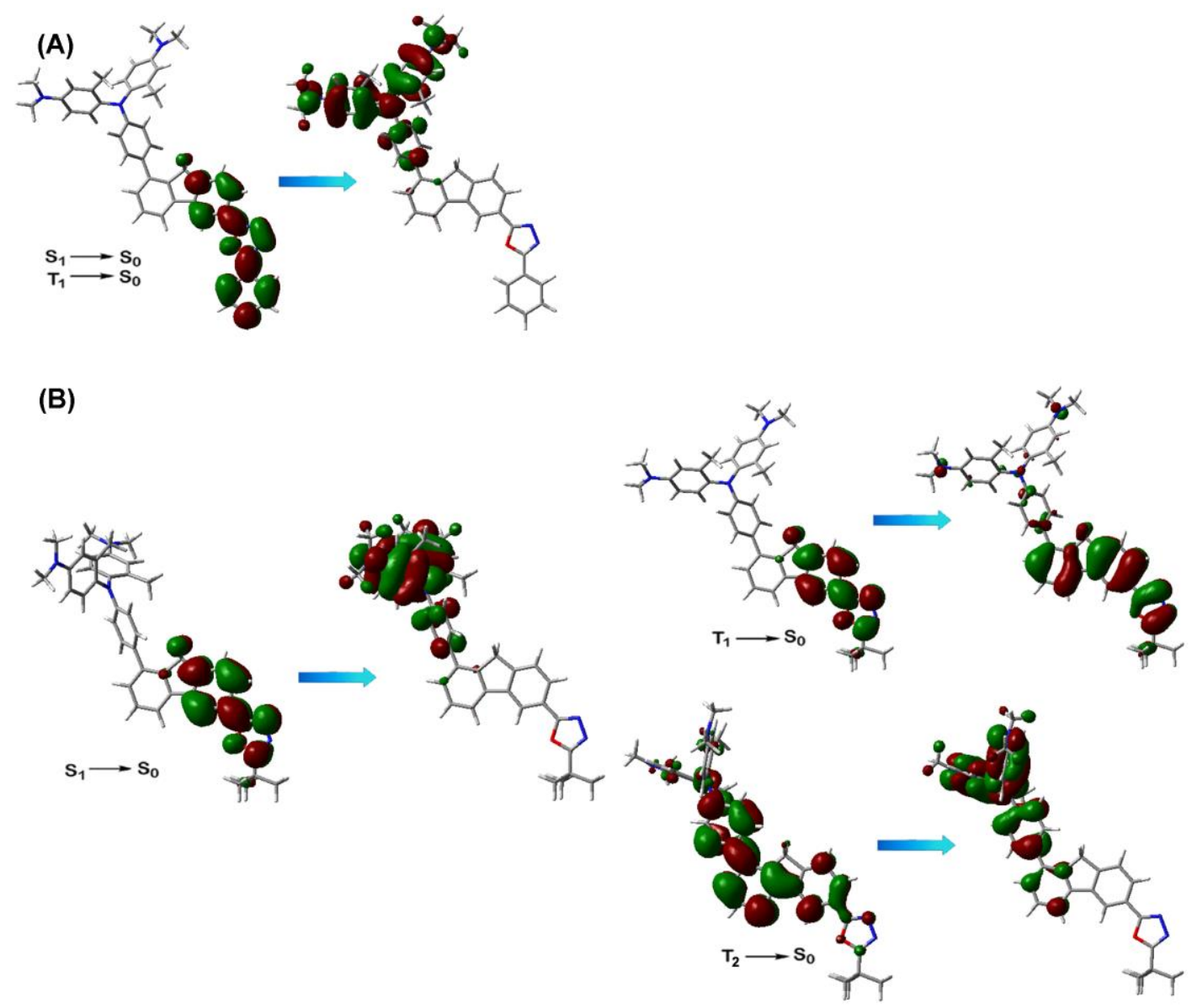

Figure S10. Molecular orbitals taking part in the emission transitions of the molecule: (A) pDMADMTPhA-m,m-Fl-MPhOx; (B) p-DMADMTPhA-m,m-Fl-OxtBu

\section{Clarification of Stokes shifts in molecule 17:}

Explanation of the values varying from $0.78 \mathrm{eV}$ to $0.89 \mathrm{eV}$ is specific geometrical variation of the molecule, which is only partially similar to that in the original system. In the lowest excited singlet both the donor and the acceptor fragments change - the outer phenyl rings of the donor flatten significantly (by ca. $20^{\circ}$ each) and delocalize more extensively, and the oxadiazole ring of the acceptor conjugates with one of the bridge phenyls. The increased planarity within the donor is partially compensated by enhanced twist to the spacer (from ca. $16^{\circ}$ in the ground state to $55^{\circ}$ in $S_{1}$ ) but this is not enough to pay off for the extended delocalization in the donor and from the acceptor to the spacer. The red shift in the excited triplet state can be attributed solely to geometry variation of the donor part, while the acceptor remains unaffected. Upon excitation, the donor again flattens but this time starting from one of the outer phenyl rings, extending to the connecting phenyl and also to one of the phenyls of fluorene. This extended $\pi$-delocalization results in $0.8 \mathrm{eV}$ of Stokes shift. It should be noted, however, that since the energy decrease of the excited states is due mainly to structural changes, Stokes shifts might be smaller in the solid state where some of the vibrational degrees of freedom will be restrained. 
Table S4: Energy and type of the longest-wavelength absorption transitions of the most promising TADF emitters from the study calculated with various DFT functionals and basis set 6-31G*; oscillator strengths for the singlet-singlet transitions and energy splitting between the first excited singlet and triplet states $\left(\Delta\left(\mathrm{S}_{1}-\mathrm{T}_{1}\right)\right)$ are given, too

\begin{tabular}{|c|c|c|c|c|c|c|c|}
\hline Molecule & Functional & $\mathrm{S}_{0} \rightarrow \mathrm{S}_{1}$ & $\mathrm{f}$ & Type & $\mathrm{S}_{0} \rightarrow \mathrm{T}_{1}$ & Type & $\Delta\left(\mathrm{S}_{1}-\mathrm{T}_{1}\right)$ \\
\hline \multirow{9}{*}{$\begin{array}{l}\text { p- } \\
\text { DMADMTPhA- } \\
\text { m,m-Fl-MPhOx }\end{array}$} & B3LYP & 2.741 & 0.0048 & $\mathrm{D}+\mathrm{X} \rightarrow \mathrm{A}+\mathrm{X}$ & 2.723 & $\mathrm{D}+\mathrm{X} \rightarrow \mathrm{A}+\mathrm{X}$ & 0.018 \\
\hline & BHandHLYP & 4.254 & 1.1036 & $D+x \rightarrow X+D+a$ & 2.205 & $\mathrm{X}+\mathrm{D}+\mathrm{A} \rightarrow \mathrm{X}+\mathrm{D}$ & 2.049 \\
\hline & M06-2X & 4.125 & 1.0426 & $D+x \rightarrow X+A+d$ & 3.272 & $A+X+d \rightarrow A+X$ & 0.835 \\
\hline & M06-HF & 4.602 & 1.5019 & $D+x \rightarrow X+A+d$ & 3.692 & $\mathrm{~A}+\mathrm{X}+\mathrm{d} \rightarrow \mathrm{A}+\mathrm{X}$ & 0.910 \\
\hline & LC-wPBE & 4.575 & 1.4961 & $D+x \rightarrow X+A+d$ & 2.260 & $\mathrm{X}+\mathrm{D}+\mathrm{A} \rightarrow \mathrm{X}+\mathrm{D}$ & 2.315 \\
\hline & wB97XD & 4.287 & 1.4047 & $D+x \rightarrow X+D+a$ & 2.823 & $\mathrm{X}+\mathrm{A}+\mathrm{d} \rightarrow \mathrm{A}+\mathrm{X}$ & 1.464 \\
\hline & CAM-B3LYP & 4.198 & 1.2356 & $D+x \rightarrow X+D+a$ & 2.657 & $\mathrm{~A}+\mathrm{X}+\mathrm{d} \rightarrow \mathrm{A}+\mathrm{X}$ & 1.541 \\
\hline & PBE0 & 2.996 & 0.0068 & $\mathrm{D}+\mathrm{x} \rightarrow \mathrm{A}+\mathrm{X}$ & 2.657 & $\mathbf{X}+\mathbf{A} \rightarrow \mathbf{A}+\mathbf{X}$ & 0.339 \\
\hline & BMK & 3.816 & 0.0935 & $\mathrm{D}+\mathrm{X} \rightarrow \mathrm{A}+\mathrm{X}$ & 3.029 & $\mathbf{X}+\mathbf{A} \rightarrow \mathbf{A}+\mathbf{X}$ & 0.787 \\
\hline \multirow{9}{*}{$\begin{array}{l}\text { p- } \\
\text { DMADMTPhA- } \\
\text { m,m-Fl-OxtBu }\end{array}$} & B3LYP & 3.077 & 0.0097 & $\mathrm{D}+\mathrm{X} \rightarrow \mathrm{A}+\mathrm{X}$ & $2.837^{\# \#}$ & $\mathrm{D}+\mathrm{X}+\mathrm{A} \rightarrow \mathrm{X}+\mathrm{D}+\mathrm{A}$ & 0.240 \\
\hline & BHandHLYP & 4.330 & 0.9355 & $D+x \rightarrow X+D+a$ & 2.271 & $\mathrm{X}+\mathrm{D}+\mathrm{a} \rightarrow \mathrm{X}+\mathrm{D}$ & 2.059 \\
\hline & M06-2X & 4.192 & 0.9450 & $D+x \rightarrow X+D+a$ & 3.370 & $\mathrm{D}+\mathrm{x} \rightarrow \mathrm{X}+\mathrm{D}+\mathrm{a}$ & 0.822 \\
\hline & M06-HF & 4.650 & 1.3007 & $D+x \rightarrow X+D+a$ & 3.749 & $\mathrm{D}+\mathrm{x} \rightarrow \mathrm{X}+\mathrm{D}+\mathrm{a}$ & 0.901 \\
\hline & LC-wPBE & 4.626 & 1.1068 & $D+x \rightarrow X+D+a$ & 2.297 & $X+A+d \rightarrow A+X$ & 2.329 \\
\hline & wB97XD & 4.348 & 1.0384 & $D+x \rightarrow X+D+a$ & 2.900 & $\mathrm{X}+\mathrm{A}+\mathrm{d} \rightarrow \mathrm{A}+\mathrm{X}$ & 1.448 \\
\hline & CAM-B3LYP & 4.263 & 1.0149 & $D+x \rightarrow X+D+a$ & 2.726 & $\mathrm{X}+\mathrm{A}+\mathrm{d} \rightarrow \mathrm{A}+\mathrm{X}$ & 1.537 \\
\hline & PBE0 & 3.334 & 0.0133 & $\mathrm{D}+\mathrm{x} \rightarrow \mathrm{A}+\mathrm{X}$ & 2.744 & $\mathbf{X}+\mathbf{A} \rightarrow \mathbf{A}+\mathbf{X}$ & 0.590 \\
\hline & BMK & 3.997 & 0.6638 & $\mathrm{D}+\mathrm{X} \rightarrow \mathrm{A}+\mathrm{X}$ & 3.126 & $\mathrm{X}+\mathrm{A} \rightarrow \mathrm{A}+\mathrm{X}$ & 0.871 \\
\hline
\end{tabular}


Table S5: Energy and type of the longest-wavelength absorption transitions of the most promising TADF emitters from the study calculated with B3LYP/6-31G* and PBE0/6-31G* with and without the Tamm-Dancoff approximation (TDA); oscillator strengths for the singletsinglet transitions and vertical energy splitting between the first excited singlet and triplet states $\left(\Delta\left(\mathrm{S}_{1}-\mathrm{T}_{1}\right)\right)$ are given, too

\begin{tabular}{|c|c|c|c|c|c|c|c|c|}
\hline Molecule & $T D A$ & Functional & $\mathrm{S}_{0} \rightarrow \mathrm{S}_{1}$ & $\mathrm{f}$ & Type & $\mathrm{S}_{0} \rightarrow \mathrm{T}_{1}$ & Type & $\Delta\left(\mathrm{S}_{1}-\mathrm{T}_{1}\right)$ \\
\hline \multirow{4}{*}{$\begin{array}{l}\mathrm{p}- \\
\text { DMADMTPhA- } \\
\mathrm{m}, \mathrm{m}-\mathrm{Fl}-\mathrm{MPhOx}\end{array}$} & \multirow{2}{*}{ No } & B3LYP & 2.741 & 0.0048 & $\mathrm{D}+\mathrm{X} \rightarrow \mathrm{A}+\mathrm{X}$ & 2.723 & $\mathrm{D}+\mathrm{X} \rightarrow \mathrm{A}+\mathrm{X}$ & 0.018 \\
\hline & & PBE0 & 2.996 & 0.0068 & $\mathrm{D}+\mathrm{X} \rightarrow \mathrm{A}+\mathrm{X}$ & 2.657 & $\mathbf{X}+\mathbf{A} \rightarrow \mathbf{A}+\mathbf{X}$ & 0.339 \\
\hline & \multirow{2}{*}{ Yes } & B3LYP & 2.742 & 0.0049 & $\mathrm{D}+\mathrm{x} \rightarrow \mathrm{A}+\mathrm{X}$ & 2.733 & $\mathrm{D}+\mathrm{x} \rightarrow \mathrm{A}+\mathrm{X}$ & 0.009 \\
\hline & & PBE0 & 2.997 & 0.0067 & $\mathrm{D}+\mathrm{x} \rightarrow \mathrm{A}+\mathrm{X}$ & 2.960 & $\mathrm{D}+\mathrm{x} \rightarrow \mathrm{A}+\mathrm{X}$ & 0.037 \\
\hline \multirow{4}{*}{$\begin{array}{l}\text { p- } \\
\text { DMADMTPhA- } \\
\text { m,m-Fl-OxtBu }\end{array}$} & \multirow{2}{*}{ No } & B3LYP & 3.077 & 0.0097 & $\mathrm{D}+\mathrm{X} \rightarrow \mathrm{A}+\mathrm{X}$ & $2.837^{\# \#}$ & $\mathrm{D}+\mathrm{X}+\mathrm{A} \rightarrow \mathrm{X}+\mathrm{D}+\mathrm{A}$ & 0.240 \\
\hline & & PBE0 & 3.334 & 0.0133 & $\mathrm{D}+\mathrm{x} \rightarrow \mathrm{A}+\mathrm{X}$ & $2.744^{\& \&}$ & $\mathbf{X}+\mathbf{A} \rightarrow \mathbf{A}+\mathbf{X}$ & 0.590 \\
\hline & \multirow{2}{*}{ Yes } & B3LYP & 3.078 & 0.0090 & $\mathrm{D}+\mathrm{x} \rightarrow \mathrm{A}+\mathrm{X}$ & $2.984^{\#}$ & $\mathrm{D}+\mathrm{x} \rightarrow \mathrm{X}+\mathrm{D}+\mathbf{a}$ & 0.094 \\
\hline & & PBE0 & 3.335 & 0.0109 & $\mathrm{D}+\mathrm{x} \rightarrow \mathrm{A}+\mathrm{X}$ & $3.054^{\&}$ & $\mathrm{D}+\mathrm{X} \rightarrow \mathrm{X}+\mathrm{D}+\mathbf{a}$ & 0.281 \\
\hline
\end{tabular}

\#\# There is a second excited triplet at energy $2.958 \mathrm{eV} ; \Delta\left(\mathrm{S}_{1}-\mathrm{T}_{2}\right)=0.119 \mathrm{eV} ; \Delta\left(\mathrm{T}_{1}-\mathrm{T}_{2}\right)=0.121 \mathrm{eV}$

\# There is a second excited triplet at energy $3.077 \mathrm{eV} ; \Delta\left(\mathrm{S}_{1}-\mathrm{T}_{2}\right)=0.001 \mathrm{eV} ; \Delta\left(\mathrm{T}_{1}-\mathrm{T}_{2}\right)=0.093 \mathrm{eV}$

$\& \&$ There are four excited triplets at energy $2.923,3.175,3.278$, and $3.328 \mathrm{eV}$;

${ }^{\&}$ There are two excited triplets at energy $3.188 \mathrm{eV}$ and $3.328 \mathrm{eV} ; \Delta\left(\mathrm{S}_{1}-\mathrm{T}_{3}\right)=0.007 \mathrm{eV} ; \Delta\left(\mathrm{T}_{1}-\mathrm{T}_{2}\right)=0.134$ $\mathrm{eV} ; \Delta\left(\mathrm{T}_{2}-\mathrm{T} 3\right)=0.140 \mathrm{eV}$

Table S6: Energy and type of the longest-wavelength emission transitions of the most promising TADF emitters from the study calculated with B3LYP/6-31G* with and without the TammDancoff approximation (TDA); oscillator strengths for the singlet-singlet transitions and vertical energy splitting between the optimized first excited singlet and triplet states $\left(\Delta\left(\mathrm{S}_{1}-\mathrm{T}_{1}\right)\right)$ are given, too

\begin{tabular}{|l|c|c|c|c|c|c|c|}
\hline Molecule & $\boldsymbol{T D A}$ & $\mathrm{S}_{1} \rightarrow \mathrm{S}_{0}$ & $\mathrm{f}$ & Type & $\mathrm{T}_{1} \rightarrow \mathrm{S}_{0}$ & Type & $\Delta\left(\mathrm{S}_{1}-\mathrm{T}_{1}\right)$ \\
\hline \hline $\begin{array}{l}\text { p- } \\
\begin{array}{l}\text { DMADMTPhA- } \\
\text { m,m-Fl-MPhOx }\end{array}\end{array}$ & Ye & $\mathbf{1 . 9 5 2}$ & $\mathbf{0 . 0 0 1 9}$ & $\mathbf{A}+\mathbf{X} \rightarrow \mathbf{D}+\mathbf{x}$ & $\mathbf{1 . 9 5 2}$ & $\mathbf{A}+\mathbf{X} \rightarrow \mathbf{D + x}$ & $\mathbf{0 . 0 0 0}$ \\
\cline { 2 - 8 } & 1.951 & 0.0020 & $\mathbf{A}+\mathbf{X} \rightarrow \mathbf{D}+\mathbf{x}$ & 1.952 & $\mathbf{A}+\mathbf{X} \rightarrow \mathbf{D + x}$ & -0.001 \\
\hline $\begin{array}{l}\text { p- } \\
\begin{array}{l}\text { DMADMTPhA- } \\
\text { m,m-Fl-OxtBu }\end{array}\end{array}$ & No & $\mathbf{2 . 1 9 1}$ & $\mathbf{0 . 0 0 6 5}$ & $\mathbf{A}+\mathbf{X} \rightarrow \mathbf{D}+\mathbf{x}$ & $\begin{array}{c}\mathbf{2 . 0 3 3} \\
\mathbf{2 . 0 3 8}\end{array}$ & $\begin{array}{c}\mathbf{A}+\mathbf{X} \rightarrow \mathbf{A}+\mathbf{X}+\mathbf{d} \\
\mathbf{X}+\mathbf{D}+\mathbf{a} \rightarrow \mathbf{D}+\mathbf{X}\end{array}$ & $\mathbf{0 . 1 5 8}$ \\
\cline { 2 - 8 } & Yes & 2.192 & 0.0067 & $\mathbf{A}+\mathbf{X} \rightarrow \mathbf{D}+\mathbf{x}$ & $2.153^{\#}$ & $\mathbf{X}+\mathbf{D}+\mathbf{a} \rightarrow \mathbf{D}+\mathbf{x}$ & 0.039 \\
\hline
\end{tabular}

\#\# There is a third excited CT triplet at $2.195 \mathrm{eV} ; \Delta\left(\mathrm{S}_{1}-\mathrm{T}_{3}\right)=-0.004 \mathrm{eV} ; \Delta\left(\mathrm{T}_{2}-\mathrm{T}_{3}\right)=0.157 \mathrm{eV}$

\# There is a second excited triplet at energy $2.192 \mathrm{eV} ; \Delta\left(\mathrm{S}_{1}-\mathrm{T}_{2}\right)=0.000 \mathrm{eV} ; \Delta\left(\mathrm{T}_{1}-\mathrm{T}_{2}\right)=0.039 \mathrm{eV}$ 
Full citation of Gaussian 09:

Gaussian 09, Revision D.01 Frisch, M. J.; Trucks, G. W.; Schlegel, H. B.; Scuseria, G. E.; Robb, M. A.; Cheeseman, J. R.; Scalmani, G.; Barone, V.; Mennucci, B.; Petersson, G. A.; Nakatsuji, H.; Caricato, M.; Li, X.; Hratchian, H. P.; Izmaylov, A. F.; Bloino, J.; Zheng, G.; Sonnenberg, J. L.; Hada, M.; Ehara, M.; Toyota, K.; Fukuda, R.; Hasegawa, J.; Ishida, M.; Nakajima, T.; Honda, Y.; Kitao, O.; Nakai, H.; Vreven, T.; Montgomery, Jr., J. A.; Peralta, J. E.; Ogliaro, F.; Bearpark, M.; Heyd, J. J.; Brothers, E.; Kudin, K. N.; Staroverov, V. N.; Kobayashi, R.; Normand, J.; Raghavachari, K.; Rendell, A.; Burant, J. C.; Iyengar, S. S.; Tomasi, J.; Cossi, M.; Rega, N.; Millam, N. J.; Klene, M.; Knox, J. E.; Cross, J. B.; Bakken, V.; Adamo, C.; Jaramillo, J.; Gomperts, R.; Stratmann, R. E.; Yazyev, O.; Austin, A. J.; Cammi, R.; Pomelli, C.; Ochterski, J. W.; Martin, R. L.; Morokuma, K.; Zakrzewski, V. G.; Voth, G. A.; Salvador, P.; Dannenberg, J. J.; Dapprich, S.; Daniels, A. D.; Farkas, Ö.; Foresman, J. B.; Ortiz, J. V.; Cioslowski, J.; Fox, D. J., Gaussian, Inc., Wallingford CT, 2009.

Full citation of Reference 11:

Hirata, Sh.; Sakai, Y.; Masui, K.; Tanaka, H.; Lee, S. Y.; Nomura, H.; Nakamura, N.; Yasumatsu, M.; Nakanotani, H.; Zhang, Q.; Shizu, K.; Miyazaki, H.; Adachi, C. Highly Efficient Blue Electroluminescence Based on Thermally Activated Delayed Fluorescence. Nature Mater. 2015, 14, 330-336.

Full citation of Reference 24:

Huang, B.; Qi, Q.; Jiang, W.; Tang, J.; Liu, Y.; Fan, W.; Yin, Zh.; Shi, F.; Ban, X.; Xu, H. Sun, Y. Thermally Activated Delayed Fluorescence Materials Based on 3,6-Di-tert-butyl-9((phenylsulfonyl)phenyl)-9H-carbazoles. Dyes Pigm. 2014, 111, 135-144.

\section{Full citation of Reference 49:}

Sangchart, T.; Niroram, A.; Kaewpuang, T.; Prachumrak, N.; Namuangruk, S.; Sudyoadsuk, T.; Keawin, T.; Saengsuwan, S.; Jungsuttiwong, S.; Maensiri, S.; Kungwan, N.; Promarak, V. Synthesis, Physical and Electroluminescence Properties of 3,6-Dipyrenylcarbazole End Capped Oligofluorenes. RSC Adv. 2015, 5, 26569-26579.

\section{References}

S1. Valchanov, G.; Ivanova, A.; Tadjer, A.; Chercka, D.; Baumgarten, M. Tuning the Optical Absorption of Potential Blue Emitters. Org. Electron. 2013, 14, 2727-2736. 